UVX 2012, 01014 (2013)

DOI: $10.1051 /$ uvx/201301014

(C) Owned by the authors, published by EDP Sciences, 2013

\title{
Notes on attosecond pulse profile measurements with the RABBIT technique
}

\author{
T. Ruchon a and A. Camper \\ CEA-Saclay, IRAMIS, Service des Photons, Atomes et Molécules, 91191 Gif-sur-Yvette, \\ France
}

\begin{abstract}
We explicitly write down the equations at the root of attosecond pulse train characterization through the RABBIT technique. The emphasize is put on the approximations required to derive the cross correlation signal. This approach provides a tutorial and general introduction to attosecond methods and tools.
\end{abstract}

\section{INTRODUCTION}

When a pulsed laser is focused in a gas target to intensities in the $10^{14} \mathrm{~W} / \mathrm{cm}^{2}$ range, the on-axis emission of a broadband XUV radiation, called High Harmonics Generation (HHG) can be observed [1,2]. It may span spectrally several tens of eV's, generally from the visible end to about $100 \mathrm{eV}$. For laser pulses durations of 2-3 fs, or when specific gating techniques are used, it shows up as a continuous spectrum. On the contrary, for pulse durations above $5 \mathrm{fs}$, a spectral comb appears, with a spacing corresponding to the frequency of two laser photons. Such a large spectrum supports a temporal duration in the attosecond domain. A major breakthrough about HHG-based XUV sources was achieved in 2001-2003 when the first demonstrations of their spectral coherence was reported and their spectral phase derivatives measured. For the first time, experimentalists were able to reconstruct attosecond pulse profiles filtered out of this radiation. The characterization technique was based on XUV/IR cross-correlation: part of the laser pulse used to initiate the HHG process was saved to initiate two photon processes in a time of flight detector placed downstream (see Fig. 1). The temporal stability of the experimental system (typically 100 as RMS, i.e. $40 \mathrm{~nm}$ ) allowed interferometric scans of the delay between the two beams. Two approaches were developed. For continuous spectra the dressing was made intense, in the $10^{13} \mathrm{~W} / \mathrm{cm}^{2}$ range (streaking regime, see e.g. Refs. [3,4] and references therein), whereas for discrete spectra it was kept low in the $10^{11} \mathrm{~W} / \mathrm{cm}^{2}$ range (see e.g. Ref. [5]). The full quantum approach to the latter technique, called RABBIT for Reconstruction of Attosecond Burst By Interferences of Two-photons transitions, is available in Refs. [6-9]. We here derive in details a more pictorial approach based on the early works of Quéré et al. [10,11] and Mairesse [12] ${ }^{1}$. Our objective is to emphasize the principle, the good points and limits of the technique through detailed calculations, readable by non specialists. The proceeding is organized in a series of little sections introducing progressively each step of the calculation.

\section{SFA FORMULA}

All through this paragraph we consider the electric fields in the detection chamber of the electronic spectrometer. The electric field of the infrared is denoted $\vec{E}_{I R}(t, z)$ and its vector potential is denoted

\footnotetext{
ae-mail: thierry.ruchon@cea.fr

${ }^{1}$ This derivation is also available in Ref. [13].

This is an Open Access article distributed under the terms of the Creative Commons Attribution License 2.0, which permits unrestricted use, distribution, and reproduction in any medium, provided the original work is properly cited.
} 


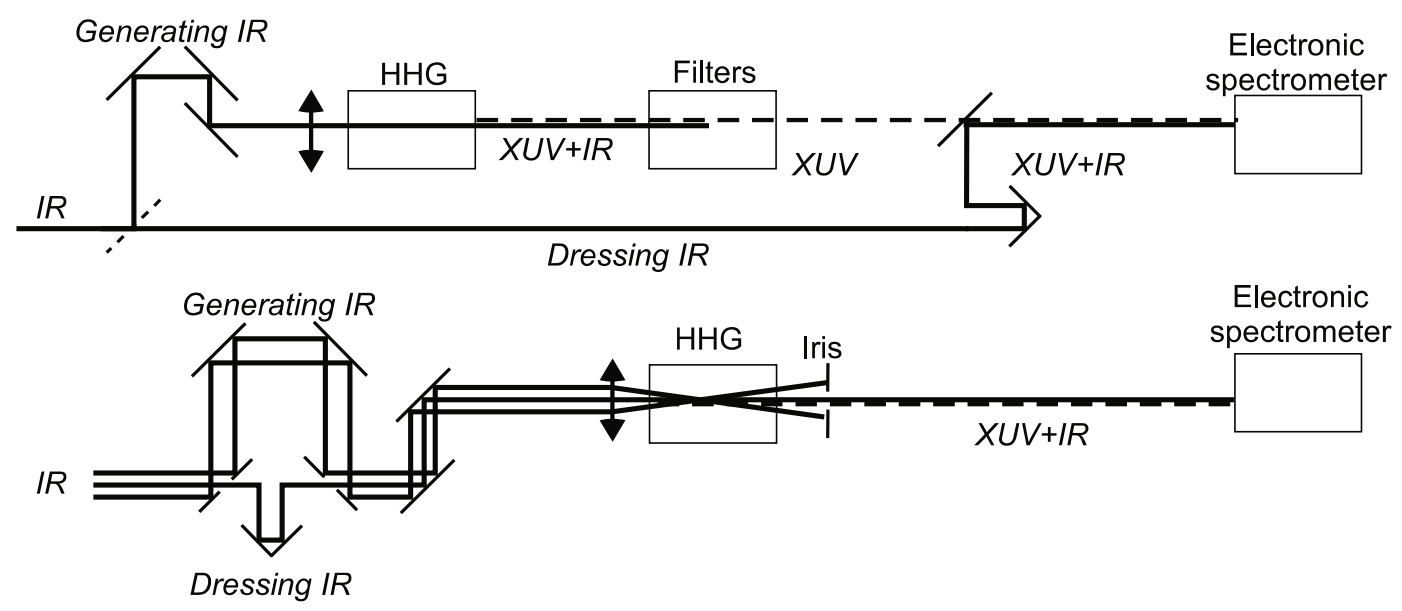

Figure 1. Two generic optical schemes to implement the RABBIT technique (The laser propagates from left to right). (top) The laser is split in two parts by a beam-splitter. The main part is used to generate harmonics which are subsequently filtered out from the remaining co-propagating infrared beam with e.g. silica plates or metallic foils. The second and weakest part $\left(\vec{E}_{I R}(t, z)\right)$ is recombined with the XUV downstream with e.g. a mirror with a hole which let through the harmonics and reflects an annular IR dressing beam. Both beams are subsequently focused in an electronic spectrometer. (bottom) Same principle but the generating IR is made annular by e.g. a drilled mirror set at $45^{\circ}$ and the weak dressing beam remains plain. They copropagate in the generating cell and are sorted out downstream by an iris set at the proper size. This latter scheme is more compact, gives in principle an absolute zero timing (see section 8), but prevents the use of plain metallic filters that would remove the dressing IR.

$\vec{E}_{I R}(t, z)=-\frac{\partial \vec{A}(t, z)}{\partial t}$ where $z$ is the abscissa along the direction of propagation of the laser. The XUV field is written $\vec{E}_{x u v}(t-\tau, z)$, where a delay $\tau$ with respect to an absolute phase has been introduced. The amplitude probability of emission of an electron which will have the final velocity $\vec{p}_{f}$ and which is located in $z$ writes $[11,14]$

$$
a\left(\vec{p}_{f}, \tau, z\right)=-i \int_{-\infty}^{\infty} d t \vec{d}\left(\vec{p}_{f}+e \vec{A}(t, z)\right) \cdot \vec{E}_{x u v}(t-\tau, z) e^{i / \hbar\left(I_{p}+\frac{p_{f}^{2}}{2 m}\right) t} e^{i \phi_{I R}\left(\vec{p}_{f}, t, z\right)}
$$

with

$$
\phi_{I R}\left(\vec{p}_{f}, t, z\right)=-\frac{1}{2 m \hbar} \int_{t}^{\infty} d t^{\prime}\left(2 e \vec{p}_{f} \cdot \vec{A}\left(t^{\prime}, z\right)+e^{2} \vec{A}\left(t^{\prime}, z\right)^{2}\right)
$$

where $\vec{d}(\vec{p})$ is the dipole amplitude of the transition from the ground state to the $\vec{p}$ state in the continuum and $\mathrm{I}_{\mathrm{p}}$ is the ionization potential of the species introduced in the spectrometer. The standard interpretation of this formula is straightforward: the atom is first ionized by the XUV field at a certain time $\mathrm{t}(\vec{d} \cdot \vec{E})$ and the freed electron subsequently flies in the IR field from time $\mathrm{t}$ to infinity, accumulating the phase due to the action (arguments of the exponential terms). This process can happen at any time, justifying the overall integration over $t$. In the following we progressively approximate this general formula to get to the main result written in Eq. (30).

\section{SLOWLY VARYING ENVELOPPE}

In all practical cases, the dressing beam is part of the infrared laser used for the generation, which has a duration in the tens of femtoseconds range when considering the generation of discrete XUV spectra (see Fig. 1). With such a duration, the slowly varying envelope approximation may be used for the IR 


\section{UVX 2012}

field. The electric field of angular frequency $\omega$ is written ${ }^{2}$

$$
\vec{E}(t, z)=E_{0}(t, z) \sin \left(\omega t-k_{0} z\right) \vec{e}
$$

where $\vec{e}$ is the direction of polarization, supposed to be linear, and $\vec{E}_{0}(t, z)=\vec{E}_{0}\left(t-z / v_{g}\right)$, $v_{g}$ being the group velocity of the fundamental. The slowly varying envelope approximation gives $\partial E_{0} / \partial t \ll E_{0} \omega$ and the associated vector potential reads

$$
\vec{A}(t, z) \simeq \frac{E_{0}(t, z)}{\omega} \cos \left(\omega t-k_{0} z\right) \vec{e} .
$$

The ponderomotive energy is defined by

$$
U_{p}(t, z)=\frac{e^{2} E_{0}(t, z)^{2}}{4 m \omega^{2}} .
$$

Introducing this approximation, Eq. (2) rewrites

$\phi_{I R}\left(\vec{p}_{f}, t, z\right)=-\frac{1}{2 m \hbar} \int_{t}^{\infty} d t^{\prime}\left(2 e\left(\vec{p}_{f} \cdot \vec{e}\right) \frac{E_{0}\left(t^{\prime}, z\right)}{\omega} \cos \left(\omega t^{\prime}-k_{0} z\right)+e^{2} \frac{E_{0}(t, z)^{2}}{\omega^{2}} \cos ^{2}\left(\omega t^{\prime}-k_{0} z\right)\right)$.

For the first term of the right hand side, an integration by parts leads to

$$
\begin{aligned}
\phi_{I R}^{(1)}\left(\vec{p}_{f}, t, z\right) & =-\frac{e}{m \hbar}\left(\vec{p}_{f} \cdot \vec{e}\right)\left(\left[\frac{E_{0}\left(t^{\prime}, z\right)}{\omega^{2}} \sin \left(\omega t^{\prime}-k_{0} z\right)\right]_{t}^{\infty}-\int_{t}^{\infty} d t^{\prime}\left(\frac{\partial E_{0}\left(t^{\prime}, z\right)}{\omega^{2} \partial t} \sin \left(\omega t^{\prime}-k_{0} z\right)\right)\right) \\
& \simeq \frac{e}{m \hbar}\left(\vec{p}_{f} \cdot \vec{e}\right) \frac{E_{0}(t, z)}{\omega^{2}} \sin \left(\omega t-k_{0} z\right) .
\end{aligned}
$$

The final line is obtained i) supposing that the field vanishes for infinite times and ii) noting that, introducing the slowly varying envelope approximation, the remaining integral is dominated by $\int_{t}^{\infty} d t^{\prime} \frac{E_{0}\left(t^{\prime}, z\right)}{\omega} \cos \left(\omega t^{\prime}-k_{0} z\right)$, which is of the same order as the calculated integral itself and can therefore be neglected.

The same procedure for the second term of the right hand side of Eq. (6) gives

$$
\begin{aligned}
\phi_{I R}^{(2)}\left(\vec{p}_{f}, t, z\right)= & -\frac{e^{2}}{4 m \hbar \omega^{2}}\left(\int_{t}^{\infty} d t^{\prime} E_{0}\left(t^{\prime}, z\right)^{2}+\left[\frac{E_{0}\left(t^{\prime}, z\right)^{2}}{2 \omega} \sin \left(2 \omega t^{\prime}-2 k_{0} z\right)\right]_{t}^{\infty}\right. \\
& \left.-\int_{t}^{\infty} d t^{\prime}\left(\frac{\partial E_{0}\left(t^{\prime}, z\right)^{2}}{2 \omega \partial t} \sin \left(2 \omega t^{\prime}-2 k_{0} z\right)\right)\right)
\end{aligned}
$$

which is approximated by

$$
\begin{aligned}
\phi_{I R}^{(2)}\left(\vec{p}_{f}, t, z\right) & \simeq-\frac{e^{2}}{4 m \hbar \omega^{2}}\left(\int_{t}^{\infty} d t^{\prime} E_{0}\left(t^{\prime}, z\right)^{2}-\frac{E_{0}(t, z)^{2}}{2 \omega} \sin \left(2 \omega t-2 k_{0} z\right)\right) \\
& \simeq-\frac{1}{\hbar} \int_{t}^{\infty} d t^{\prime} U_{p}\left(t^{\prime}, z\right)+\frac{1}{2 \hbar \omega} U_{p}(t, z) \sin \left(2 \omega t-2 k_{0} z\right) .
\end{aligned}
$$

In the following we introduce these expressions of the phase (Eqs. (7) and (10)) in Eq. (1) and simplify it introducing a Bessel expansion of the phase.

\footnotetext{
2 The phase is chosen to be zero. On the contrary, a delay $\tau$ was introduced on the XUV field to take into account the relative phase between the two fields.
} 


\section{Web of Conferences}

\section{GENERALIZED BESSEL FUNCTIONS}

From Ref. [15], the generalized Bessel functions $J_{n}^{(p, q)}(u, v)$ are defined by

$$
e^{u \sin (p t)+v \sin (q t)}=\sum_{n=-\infty}^{\infty} J_{n}^{(p, q)}(u, v) e^{i n t}
$$

$e^{i \phi_{I R}}$ has some $\omega$ and $2 \omega$ oscillations. Setting the parameters

$$
\left\{\begin{aligned}
p & =1 \\
q & =2 \\
u\left(t, z, \vec{p}_{f}\right) & =\frac{e}{m \hbar} \frac{E_{0}(t, z)}{\omega^{2}}\left(\vec{p}_{f} \cdot \vec{e}\right)=2 \sqrt{2} \sqrt{\frac{U_{p}(t, z)}{\hbar \omega}} \sqrt{\frac{E_{f}}{\hbar \omega}} \\
v(t, z) & =\frac{1}{2 \hbar \omega} U_{p}(t, z)
\end{aligned}\right.
$$

where we have introduced the final energy of the electron given by $E_{f}=p_{f}^{2} / 2 m$, we get

$$
e^{i \phi_{I R}}=e^{i \phi_{p}(t, z)} \sum_{n=-\infty}^{\infty} J_{n}^{(1,2)}\left(u\left(t, z, \vec{p}_{f}\right), v(t, z)\right) e^{i n \omega(t-z / c)},
$$

where

$$
e^{i \phi_{p}(t, z)}=e^{-\frac{i}{\hbar} \int_{t}^{\infty} d t^{\prime} U_{p}\left(t^{\prime}, z\right)} .
$$

$\phi_{p}(t, z)$ is called the ponderomotive phase. Note that here $\phi_{p}(t, z), u\left(t, z, \vec{p}_{f}\right)$ and $v(t, z)$ only include the envelope and are therefore slowly varying with $t$. Besides, it should be noted that $v$ is the ponderomotive shift in photon energy units, which is small in the RABBIT regime. For instance, for an intensity of $10^{11} \mathrm{~W} / \mathrm{cm}^{2}, U_{p}=6 \mathrm{meV}$ and $v=410^{-3}$. Since usually the final energies of the electrons detected are of the order of a few tens of photon energy, $v$ will automatically remain small if $u \ll 1$. Again, with $\mathrm{I}=10^{11} \mathrm{~W} / \mathrm{cm}^{2}$, and a final energy of the electron of $10 \mathrm{eV}, u \simeq 0.4$, which is a small value at the scale of the variations of the Bessel functions plotted in Fig. 2. Introducing this expansion in Eq. (1) we get

$$
\begin{aligned}
a\left(\vec{p}_{f}, \tau, z\right)= & -i \sum_{n} \int_{-\infty}^{\infty} d t \vec{d}\left(\vec{p}_{f}+e \vec{A}(t, z)\right) \cdot \vec{E}_{x u v}(t-\tau, z) e^{i / \hbar\left(I_{p}+\frac{p_{f}^{2}}{2 m}\right) t} \\
& \times e^{i \phi_{p}(t, z)} J_{n}^{(1,2)}\left(u\left(t, z, \vec{p}_{f}\right), v(t, z)\right) e^{i n \omega(t-z / c)} .
\end{aligned}
$$

For small electric fields so that $u, v \ll 1$, the $J_{n}^{(1,2)}$ are smaller and smaller as $n$ increases and the first one, $J_{0}^{(1,2)} \simeq 1$ (see Fig. 2). So the sum, in a first approximation can be truncated to $n=0$ and $n= \pm 1^{3}$. The $0^{\text {th }}$ order term writes

$$
a^{0}\left(\vec{p}_{f}, \tau . z\right)=-i \int_{-\infty}^{\infty} d t J_{0}^{(1,2)}\left(u\left(t, z, \vec{p}_{f}\right), v(t, z)\right) \vec{d}\left(\vec{p}_{f}+e \vec{A}(t, z)\right) \cdot \vec{E}_{x u v}(t-\tau, z) e^{i / \hbar\left(I_{p}+\frac{p_{f}^{2}}{2 m}\right) t+i \phi_{p}(t, z)}
$$

and the $n= \pm 1$

$$
\begin{aligned}
a^{ \pm 1}\left(\vec{p}_{f}, \tau, z\right)= & -i \int_{-\infty}^{\infty} d t J_{ \pm 1}^{(1,2)}\left(u\left(t, z, \vec{p}_{f}\right), v(t, z)\right) \vec{d}\left(\vec{p}_{f}+e \vec{A}(t, z)\right) \cdot \vec{E}_{x u v}(t-\tau, z) \\
& \times e^{i / \hbar\left(I_{p}+\frac{p_{f}^{2}}{2 m}\right) t+i \phi_{p}(t, z) \pm i \omega(t-z / c)} .
\end{aligned}
$$

\footnotetext{
3 This means that there is no third order or higher order sidebands as will be seen below. Ref. [16] reports a thorough study of the transition from the RABBIT regime to the regime in which higher order sidebands need to be considered. In summary, if the dressing is too intense, the RABBIT analysis turns wrong and an experimental criterion to check the regime is to verify that the sidebands are oscillating with just a $2 \omega$ period, no higher frequencies.
} 

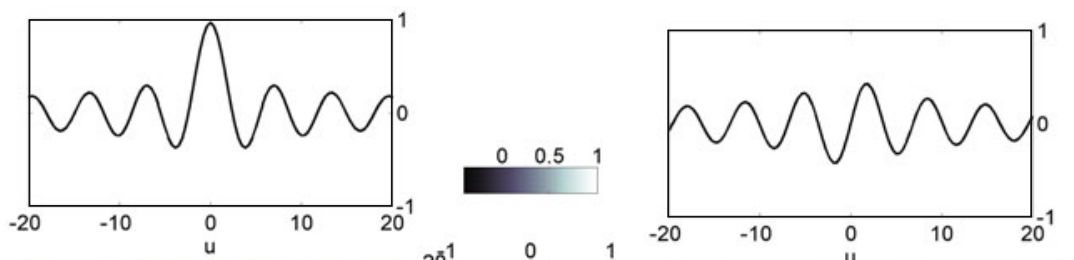

$\begin{array}{rrr}-0.5 & 0 & 0.5\end{array}$
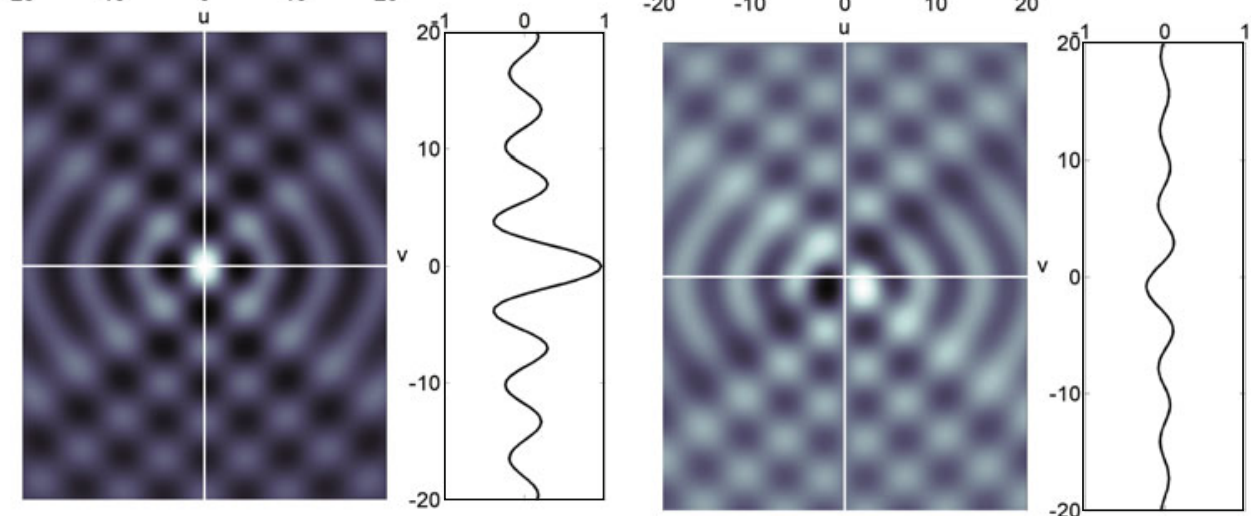

Figure 2. Generalized Bessel functions $J_{0}^{(1,2)}(u, v)$ (left) and $J_{1}^{(1,2)}(u, v)$ (right) along with the cut throughs the origin.

\section{DISTRIBUTION PROBABILITIES}

The probability density, $\mathcal{P}(E, \tau, z)$, of finding an electron with energy $E$, is proportional to $|a(\sqrt{2 m E}, \tau, z)|^{2}$, which writes, using Eq. (15)

$$
\mathcal{P}(E, \tau, z)=\left|a^{0}(\sqrt{2 m E}, \tau, z)+a^{1}(\sqrt{2 m E}, \tau, z)+a^{-1}(\sqrt{2 m E}, \tau, z)+\ldots\right|^{2}
$$

Using the previous formulas, and restricting again to the zeroth and first order, we get

$$
\begin{aligned}
a^{0}(\sqrt{2 m E}, \tau, z)= & -i \int_{-\infty}^{\infty} d t J_{0}^{(1,2)}(u(t, z, \sqrt{2 m E}), v(t, z)) \vec{d}(\sqrt{2 m E}+e \vec{A}(t, z)) \\
& \times \vec{E}_{x u v}(t-\tau, z) e^{i \frac{\left(I_{p}+E\right)}{\hbar} t+i \phi_{p}\left(t, z^{\prime}\right)}
\end{aligned}
$$

and

$$
\begin{aligned}
a^{ \pm 1}(\sqrt{2 m E}, \tau, z)= & -i \int_{-\infty}^{\infty} d t J_{ \pm 1}^{(1,2)}(u(t, z, \sqrt{2 m E}), v(t, z)) \vec{d}(\sqrt{2 m E}+e \vec{A}(t, z)) \\
& \times \vec{E}_{x u v}(t-\tau, z) e^{i \frac{\left(I_{p}+E \pm h v\right)}{\hbar} t+i \phi_{p}\left(t, z^{\prime}\right) \mp i \omega z / c}
\end{aligned}
$$

The two formulas are very similar but for a shift of $\pm h v$ on the energy scale. This is where the sidebands come from as will be made clearer later on. The probability density will write

$$
\mathcal{P}(E, \tau, z)=\left|a^{0}\right|^{2}+\left|a^{1}\right|^{2}+\left|a^{-1}\right|^{2}+2 \Re\left(a^{0} a^{1 *}\right)+2 \Re\left(a^{0} a^{-1 *}\right)+2 \Re\left(a^{1} a^{-1 *}\right)+\mathcal{O}\left(a_{1}^{2}\right)
$$




\section{Web of Conferences}

\section{ATTOSECOND PULSES AS A SUM OF HARMONICS}

To simplify further the above formulas 19-20, we successively scrutinize each term of the integral.

As for the XUV field, we will consider a sum of odd harmonics, linearly and identically polarized and discard the vectorial notation:

$$
E_{x u v}(t)=\sum_{q \text { odd }} A_{q} e^{i q \omega\left(t-z / v_{q}\right)+\Psi_{q}} \vec{e} .
$$

For the dipole element, we reckon that the energy associated to the vector potential (the ponderomotive energy) is small compared to the final energy of the electrons - which may be some tens of eVs, and that the cross sections are usually smooth across this kind of energy scale. For these small "shears", we thus write

$$
\vec{d}\left(\sqrt{2 m E}+e \vec{A}\left(t_{k}+\tau, z\right)\right) \simeq \vec{d}(\sqrt{2 m E}) .
$$

As for the Bessel functions, they vary slowly since they only include some envelope terms. If restricting the scan to reasonable delays of a few periods the time dependence can be removed.

Finally, the "ponderomotive" phase, defined in Eq. 14, is approximated at first order by a linear function of time: $\phi_{p}(t+\tau, z) \simeq \phi_{p}^{0}(\tau, z)+U_{p}(\tau, z) / \hbar \times t$. This is valid around the top of the pulse.

Plugging these expressions in Eq. 19 and 20, we get

$$
\begin{aligned}
a^{0}(\sqrt{2 m E}, \tau, z)= & -i J_{0}^{(1,2)}(u, v) \vec{d}(\sqrt{2 m E}) \cdot \vec{e} \sum_{q \text { odd }} A_{q} \int_{-\infty}^{\infty} d t e^{i q \omega\left(t-\tau-z / v_{q}\right)+i \Psi_{q}} e^{i / \hbar\left(I_{p}+E+U_{p}(\tau)\right) t+i \phi_{p}^{0}(\tau, z)} \\
= & -i J_{0}^{(1,2)}(u, v) \vec{d}(\sqrt{2 m E}) \cdot \vec{e} \sum_{q \text { odd }} A_{q} e^{i q \omega\left(-\tau-z / v_{q}\right)+i \Psi_{q}+i \phi_{p}^{0}(\tau, z)} \\
& \times \int_{-\infty}^{\infty} d t e^{i / \hbar\left(q \omega \hbar+I_{p}+E+U_{p}(\tau)\right) t} \\
= & -i J_{0}^{(1,2)}(u, v) \vec{d}(\sqrt{2 m E}) \cdot \vec{e} \sum_{q \text { odd }} A_{q} e^{i q \omega\left(-\tau-z / v_{q}\right)+i \Psi_{q}+i \phi_{p}^{0}(\tau, z)} \delta\left(q \hbar \omega+I_{p}+E+U_{p}(\tau)\right)
\end{aligned}
$$

and

$$
\begin{aligned}
a^{ \pm 1}(\sqrt{2 m E}, \tau, z)= & -i J_{ \pm 1}^{(1,2)}(u, v) \vec{d}(\sqrt{2 m E}) \cdot \vec{e} \sum_{q \text { odd }} A_{q} e^{i q \omega\left(-\tau-z / v_{q}\right)+i \Psi_{q}+i \phi_{p}^{0}(\tau, z) \mp i \omega z / c} \\
& \times \delta\left((q \pm 1) \hbar \omega+I_{p}+E+U_{p}(\tau)\right) .
\end{aligned}
$$

\section{MODULATED SPECTRUM AND SIDEBANDS}

We now examine the different terms of Eq. (21) once these approximations are introduced.

\subsection{Squared terms}

$\left|a^{0}(\sqrt{2 m E}, \tau, z)\right|^{2}$ will have some products of $\delta$ function which are non zero only when the arguments are the same. So the double sum reduces to a single sum:

$$
\left|a^{0}(\sqrt{2 m E}, \tau, z)\right|^{2}=\left|J_{0}^{(1,2)}(u, v)\right|^{2}\left|\vec{d}(\sqrt{2 m E}) \cdot \vec{e}_{x u v}\right|^{2} \sum_{q \text { odd }} A_{q} A_{q}^{*} \times \delta\left(q \hbar \omega+I_{p}+E+U_{p}(\tau)\right) .
$$




\section{UVX 2012}

Since the Bessel function is almost 1 (Fig. 2), it is a copy of the harmonic spectrum times the transition probability. Note that there is a global shift of the spectrum of $U_{p}(\tau)$ Measuring this shift is a way to measure $U_{p}$ (see e.g. [17]) and assess the validity of the approximations made here.

$\left|a^{ \pm 1}(\sqrt{2 m E}, \tau, z)\right|^{2}$ will give almost the same, but for a shift of the spectrum of one harmonic. Nevertheless, the Bessel function is small so this "background" on the sidebands will be small. It is of the order of

$\left|a^{ \pm 1}(\sqrt{2 m E}, \tau, z)\right|^{2}=\left|J_{ \pm 1}^{(1,2)}(u, v)\right|^{2}\left|\vec{d}(\sqrt{2 m E}) \cdot \vec{e}_{x u v}\right|^{2} \sum_{q \text { odd }} A_{q} A_{q}^{*} \times \delta\left((q \pm 1) \hbar \omega+I_{p}+E+U_{p}(\tau)\right)$.

\subsection{Cross terms}

The first type of cross term is $2 \Re\left(a^{0} a^{ \pm 1 *}\right)$. It takes products of $\delta$ functions of different arguments. Indeed only the odd $q$ should be considered and $a^{0}(\sqrt{2 m E}, \tau, z)$ is proportional to $\delta\left(q \hbar \omega+I_{p}+E+U_{p}(\tau)\right)$ while $a^{1}(\sqrt{2 m E}, \tau, z)$ is proportional to $\delta\left((q+1) \hbar \omega+I_{p}+E+U_{p}(\tau)\right)$. So they never match and the sum is zero ${ }^{4}$.

Now the last cross term, $2 \Re\left(a^{1} a^{-1 *}\right)$ writes

$$
\begin{array}{r}
a^{1}(\sqrt{2 m E}, \tau, z) a^{-1 *}(\sqrt{2 m E}, \tau, z)=J_{1}^{(1,2)}(u, v) \cdot J_{-1}^{(1,2)}(u, v)|\vec{d}(\sqrt{2 m E}) \cdot \vec{e}|^{2} \\
\times \sum_{q 1, q 2 \text { odd }} A_{q 1} A_{q 2}^{*} e^{-i\left(q_{1}-q_{2}\right) \omega \tau-i \omega z\left(q_{1} / v_{q 1}-q_{2} / v_{q 2}\right)-2 i \omega z / c+i \Psi_{q 1}-i \Psi_{q 2}} \\
\quad \times \delta\left(\left(q_{1}+1\right) \hbar \omega+I_{p}+E+U_{p}(\tau)\right) \delta\left(\left(q_{2}-1\right) \hbar \omega+I_{p}+E+U_{p}(\tau)\right) .
\end{array}
$$

Only $\delta q=q_{2}-q_{1}=2$ terms remain, ie for a given $q_{1}, q_{2}$ needs to be $q_{1}+2$. We thus get

$$
\begin{aligned}
& 2 \Re\left(a^{1}(\sqrt{2 m E}, \tau, z) a^{-1 *}(\sqrt{2 m E}, \tau, z)\right)=2 J_{1}^{(1,2)}(u, v) \cdot J_{-1}^{(1,2)}(u, v) \\
& \times|\vec{d}(\sqrt{2 m E}) \cdot \vec{e}|^{2} \Re \sum_{q \text { odd }} A_{q} A_{q+2}^{*} e^{+2 i \omega \tau-i \omega z\left(q / v_{q}-(q+2) / v_{q+2}\right)-2 i \omega z / c+\Psi_{q}-\Psi_{q+2}} \\
& \times \delta\left((q+1) \hbar \omega+I_{p}+E+U_{p}(\tau)\right) .
\end{aligned}
$$

From the definition of the train the $A_{q}$ 's are supposed to be real. In the time of flight, the density is low, so the dispersion as well and $v_{q} \simeq c$. So

$$
\begin{aligned}
2 \Re\left(a^{1}(\right. & \left.\sqrt{2 m E}, \tau, z) a^{-1 *}(\sqrt{2 m E}, \tau, z)\right)=2 J_{1}^{(1,2)}(u, v) \cdot J_{-1}^{(1,2)}(u, v)|\vec{d}(\sqrt{2 m E}) \cdot \vec{e}|^{2} \\
& \times \sum_{q \text { odd }} A_{q} A_{q+2} \cos \left(2 \omega \tau-\Psi_{q+2}+\Psi_{q}\right) \delta\left((q+1) \hbar \omega+I_{p}+E+U_{p}(\tau)\right) .
\end{aligned}
$$

This term sums up with the $\left|a^{ \pm 1}(\sqrt{2 m E}, \tau, z)\right|^{2}$ terms of Eq. (26). Indeed, for one given q, one get the interference term as

$$
\begin{aligned}
|\vec{d}(\sqrt{2 m E}) \cdot \vec{e}|^{2}( & \left|J_{1}^{(1,2)}(u, v) A_{q}\right|^{2}+\left|J_{-1}^{(1,2)}(u, v) A_{q-2}\right|^{2}+2 J_{1}^{(1,2)}(u, v) \cdot J_{-1}^{(1,2)}(u, v) A_{q} A_{q-2} \\
& \left.\times \cos \left(2 \omega \tau+\Psi_{q+2}-\Psi_{q}\right)\right) .
\end{aligned}
$$

Finally, for $v \ll 1$, we have $J_{1}^{(1,2)}(u, v) \simeq-J_{-1}^{(1,2)}(u, v)$ (Fig. 2). We can then factorize the previous equation as

$$
|\vec{d}(\sqrt{2 m E}) \cdot \vec{e}|^{2}\left|J_{1}^{(1,2)}(u, v)\right|^{2}\left(\left|A_{q}\right|^{2}+\left|A_{q-2}\right|^{2}+2 A_{q} A_{q-2} \cos \left(2 \omega \tau+\Psi_{q+2}-\Psi_{q}\right)\right) .
$$

\footnotetext{
4 This statement would not hold if the source was delivering odd and even harmonics as in e.g. Ref. [18].
} 


\section{Web of Conferences}

We get a very generic interference formula, from which the difference of spectral phase of two successive harmonics of the radiation $\left(\Psi_{q+2}-\Psi_{q}\right)$ may be measured. This formula is the main result of the calculation. Note that the phase of the ionization dipole canceled out. This is one of the main limit of this approach. Indeed, the full quantum calculation would introduce a two-photon matrix element going from the ground state to the final electronic state in the continuum (see e.g. Refs. [7,9, 19]). In that case, the phase of the photoionization dipole would remain in the final cosine term, just adding to $\Psi_{q+2}-\Psi_{q}$. They are therefore two ways to use the technique, focusing on retrieving one or the other phase difference.

Knowing the phase of the radiation, the analysis of the oscillations of the cosine terms gives access to the phase of the electronic wave packets emitted in this two-photon process. Though recognized from the early days [20,21], this way of using the technique recently received a renewed interest, with the perspective of accessing photoionization timings [5, 22-24].

On the contrary, if one uses a detection species having a flat or known dipole phase, the characterization of the oscillation gives access to the derivative of the spectral phase of the radiation (Group delay $\left.{ }^{5}, G D(q)=\left(\Psi_{q+2}-\Psi_{q}\right) / 2 \omega\right)$. However, it should be noted that the absolute timing of the delay between the XUV and IR fields is usually experimentally undetermined: in most schemes, just known steps are applied on a piezo delay stage with an arbitrary origin. In these cases, $G D(q)$ is determined up to a constant common to all q's, which means that it is only the Group Delay Dispersion $\left(G D D_{q}=\partial G D / \partial \omega\right)$ that is experimentally determined.

\section{ABSOLUTE TIMING}

In this final paragraph we address the question of the determination of the phase reference of the XUV vs. the IR, i.e the determination of the integration constant introduced right above. In Refs. [25-27], using a collinear setup (Fig. 1, bottom), the authors claimed to have measured this absolute timing. In these experiments the generation occurred in a medium in which the probe and the pump overlapped with a varying delay. So the total generating field was the interferences of both. Indeed, if we write one of the field $E_{0} \cos (\omega t-k z)$ and the second $\alpha E_{0} \cos \left(\omega\left(t-\tau_{0}\right)-k z\right)$, where $\tau_{0}$ is the delay, imposed from the outside by a piezo stage on the dressing beam and $\alpha \ll 1$ is a constant, the total generating field writes

$$
\begin{aligned}
\vec{E}_{\text {gen }}(t, z) & =E_{0}(t, z) \Re\left(e^{i \omega t-k z}+\alpha e^{i \omega\left(t-\tau_{0}\right)-k z}\right) \\
& =E_{0}(t, z) \Re\left(e^{i \omega t-k z}\left(1+\alpha e^{-i \omega \tau_{0}}\right)\right) \\
& \simeq E_{0}(t, z) \cos (\omega t-k z)\left(1+\alpha \cos \left(\omega \tau_{0}\right)\right) .
\end{aligned}
$$

Equation (33) is plotted in Fig. 3 for two different $\alpha$ 's. For little $\alpha$ it mostly remains a single sinus wave, with a modulated amplitude. Importantly, the phase is that of the main wave.

Now this will modulate the amplitude of the harmonics $\left(A_{q}\right.$ 's) and the generation conditions in the medium. Since the modulation is small, we can consider that the latter point is a second order effect and therefore the phase of the harmonics emitted is barely modified. The generation is non linear, and goes approximately as the $9 / 2$ th power of the intensity [26]. So $A_{q} A_{q}^{*}$ in Eq. (25) is a power of the field and a linear Taylor expansion shows that it should be replaced by $A_{q} A_{q}^{*}\left(1+\alpha^{\prime} \cos \left(\omega \tau_{0}\right)\right), \alpha^{\prime}$ being something like $9 \alpha$. So the yield is modulated with a phase $\omega \tau_{0}$. Monitoring the total yield while scanning $\tau_{0}$ gives an absolute position of the delay stage and thus the GD may be measured with no integration constant.

This procedure, though extremely attractive, seems to provide some uncontrolled systematic errors when put into practice [28]. A more precise development of the above equations and a thorough study

\footnotetext{
${ }^{5}$ Note that $\Psi_{q+2}-\Psi_{q}$ is determined modulo $2 \pi$, hence the GD modulo $1 / T$, where $\mathrm{T}$ is the period of the laser. In standard cases, this uncertainty can easily be coped with based on simple models, such as the three step model of HHG.
} 

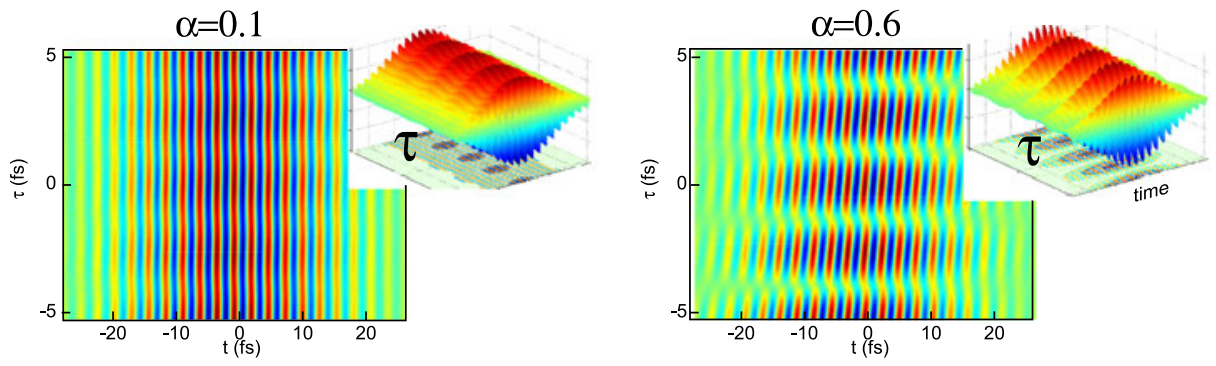

Figure 3. IR pulse profile in the generating medium as a function of the delay between the generating beam and the dressing beam $(\tau)$ for two different ratios of the amplitude (left) $\alpha=0.1$, (right) $\alpha=0.6$, in the scheme at the bottom of Fig. 1.

of the effect of some diffuse light issued from the generating beam and diffracted by the filtering iris should be initiated to assess more precisely the validity domain of this approach. To our knowledge, this still needs to be done.

\section{CONCLUSION}

We have derived the equations at the heart of the RABBIT technique, going progressively through all approximations. As a final word, we would like to emphasize that what is measured with this technique is the relative phase between electrons photoionized towards states of different final energies. This is completely different from the synchrotron measurements of the relative phases of several partial waves through different channels. Though the theory is well understood, some weak points still need to be addressed. To mention but two important ones, the relation of the measured phase to the time of photoionization seems to be of utmost current interest though not fully understood [5,22-24]. Second, the absolute determination of the phase would be extremely valuable in the context of orbital tomography [13].

\section{References}

[1] F. Krausz, M. Ivanov, Reviews of Modern Physics 81(1), 163 ( 72) (2009)

[2] K. Varjú, P. Johnsson, J. Mauritsson, A. L'Huillier, R. López-Martens, American Journal of Physics 77(5), 389 (2009)

[3] J. Gagnon, E. Goulielmakis, V. Yakovlev, Applied Physics B: Lasers and Optics 92, 25 (2008)

[4] J. Gagnon, V.S. Yakovlev, Opt. Express 17(20), 17678 (2009)

[5] M. Swoboda, T. Fordell, K. Klünder, J.M. Dahlström, M. Miranda, C. Buth, K.J. Schafer, J. Mauritsson, A. L’Huillier, M. Gisselbrecht, Phys. Rev. Lett. 104(10), 103003 (2010)

[6] V. Véniard, R. Taïeb, A. Maquet, Phys. Rev. Lett. 74, 4161 (1995)

[7] V. Véniard, R. Taïeb, A. Maquet, Phys. Rev. A 54, 721 (1996)

[8] H.G. Muller, Appl. Phys. B 74, 17 (2002)

[9] M. Dahlström, Ph.D. thesis, Lund University (2011)

[10] Y. Mairesse, F. Quéré, Phys. Rev. A 71, 011401(R) (2005)

[11] F. Quéré, Y. Mairesse, J. Itatani, J. Mod. Opt. 52, 339 (2005)

[12] Y. Mairesse, Ph.D. thesis, Université Paris Sud - Paris XI (2005)

[13] S. Haessler, Ph.D. thesis, Université Paris Sud - Paris XI (2009)

[14] M. Kitzler, N. Milosevic, A. Scrinci, T. Brabec, Phys. Rev. Lett. 88, 173904 (2002)

[15] H.J. Korsch, A. Klumpp, D. Witthaut, Journal of Physics A: Mathematical and General 39(48), 14947 (2006) 


\section{Web of Conferences}

[16] M. Swoboda, J.M. Dahlström, T. Ruchon, P. Johnsson, J. Mauritsson, A. L'Huillier, K.J. Schafer, Laser Physics 19, 1591 (2009)

[17] K. Varjú, P. Johnsson, J. Mauritsson, T. Remetter, T. Ruchon, Y. Ni, F. Lépine, M. Kling, J. Khan, K.J. Schafer et al., Journal of Physics B: Atomic, Molecular and Optical Physics 39(18), 3983 (2006)

[18] J. Mauritsson, P. Johnsson, E. Gustafsson, A. L'Huillier, K.J. Schafer, M.B. Gaarde, Phys. Rev. Lett. 97, 013001 (2006)

[19] E.S. Toma, H.G. Muller, J. Phys. B 35, 3435 (2002)

[20] P. Johnsson, R. López-Martens, S. Kazamias, J. Mauritsson, C. Valentin, T. Remetter, K. Varjú, M.B. Gaarde, Y. Mairesse, H. Wabnitz et al., Phys. Rev. Lett. 95, 013001 (2005)

[21] P. Johnsson, K. Varjú, T. Remetter, E. Gustafsson, J. Maritsson, R. López-Martens, S. Kazamias, C. Valentin, P. Balcou, M.B. Gaarde et al., J. Mod. Opt. 53, 233 (2006)

[22] K. Klünder, J.M. Dahlström, M. Gisselbrecht, T. Fordell, M. Swoboda, D. Guénot, P. Johnsson, J. Caillat, J. Mauritsson, A. Maquet et al., Physical Review Letters 106(14), 143002 (2011)

[23] S. Haessler, B. Fabre, J. Higuet, J. Caillat, T. Ruchon, P. Bréger, B. Carré, E. Constant, A. Maquet, E. Mével et al., Physical Review A 80(1), 011404 (2009)

[24] J. Caillat, A. Maquet, S. Haessler, B. Fabre, T. Ruchon, P. Salières, Y. Mairesse, R. Taïeb, Physical Review Letters 106(9), 093002 (2011)

[25] S.A. Aseyev, Y. Ni, L.J. Frasinski, H.G. Muller, M.J.J. Vrakking, Phys. Rev. Lett. 91, 223902 (2003)

[26] L.C. Dinu, H.G. Muller, S. Kazamias, G. Mullot, F. Augé, P. Balcou, P.M. Paul, M. Kovačev, P. Bréger, P. Agostini, Phys. Rev. Lett. 91, 063901 (2003)

[27] Y. Mairesse, A. de Bohan, L.J. Frasinski, H. Merdji, L.C. Dinu, P. Monchicourt, P. Breger, M. Kovačev, R. Taïeb, B. Carré et al., Science 302, 1540 (2003)

[28] S. Haessler, J. Caillat, W. Boutu, C. Giovanetti-Teixeira, T. Ruchon, T. Auguste, Z. Diveki, P. Bréger, A. Maquet, B. Carré et al., Nature Physics 6(3), 200 (2010) 